\title{
An improved quasi-Newton equation on the quasi-Newton methods for unconstrained optimizations
}

\author{
Basim A. Hassan ${ }^{1}$, Kanikar Muangchoo $^{2}$, Fadhil Alfarag ${ }^{3}$, Abdulkarim Hassan Ibrahim ${ }^{4}$ \\ Auwal Bala Abubakar ${ }^{5}$ \\ ${ }^{1}$ Department of Mathematics, College of Computers Sciences and Mathematics, University of Mosul, Iraq \\ ${ }^{2}$ Faculty of Science and Technology, Rajamangala University of Technology Phra Nakhon (RMUTP), Thailand \\ ${ }^{3}$ School of mathematics, University of Birmingham, United Kingdom \\ ${ }^{4}$ Department of Mathematics, Faculty of Science, King Mongkut's University of Technology Thonburi (KMUTT), \\ Bangkok, Thailand \\ ${ }_{5}^{5}$ Department of Mathematical Sciences, Faculty of Physical Sciences, Bayero University, Kano, Kano, Nigeria \\ ${ }^{5}$ Department of Mathematics and Applied Mathematics, Sefako Makgatho Health Sciences University, Ga-Rankuwa, \\ Pretoria, Medunsa-0204, South Africa
}

\begin{tabular}{l}
\hline \hline Article Info \\
\hline Article history: \\
Received Dec 4, 2020 \\
Revised Mar 17, 2021 \\
Accepted Apr 6, 2021
\end{tabular}

Keywords:

Global convergence property Numerical experiments Quasi-Newton equation Unconstrained optimizations

\begin{abstract}
Quasi-Newton methods are a class of numerical methods for solving the problem of unconstrained optimization. To improve the overall efficiency of resulting algorithms, we use the quasi-Newton methods which is interesting for quasi-Newton equation. In this manuscript, we present a modified BFGS update formula based on the new quasi-Newton equation, which give a new search direction for solving unconstrained optimizations proplems. We analyse the convergence rate of quasi-Newton method under some mild condition. Numerical experiments are conducted to demonstrate the efficiency of new methods using some test problems. The results indicates that the proposed method is competitive compared to the BFGS methods as it yielded fewer iteration and fewer function evaluations.
\end{abstract}

This is an open access article under the $\underline{C C B Y-S A}$ license.

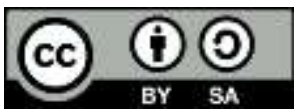

\section{Corresponding Author:}

Kanikar Muangchoo

Faculty of Science and Technology

Rajamangala University of Technology Phra Nakhon (RMUTP)

1381, Pracharat 1 Road, Wongsawang, Bang Sue, Bangkok 10800 Thailand

Email: kanikar.m@rmutp.ac.th

\section{INTRODUCTION}

Quasi-Newton method is one of the main iterative methods designed to solve unconstrained optimization problem [1]. The method has the (1).

$$
\operatorname{Min} f(x), \mathrm{x} \in \mathrm{R}^{\mathrm{n}}
$$

where $f: R^{n} \rightarrow R$, is smooth function. More details can be found in [2]. The iterative method takes the (2).

$$
x_{k+1}=x_{k}+\alpha_{k} d_{k}
$$


where $\alpha_{k}$ is the parameter determined by exact line-search as.

$$
\alpha_{k}=-\frac{g_{k}^{T} d_{k}}{d_{k}^{T} Q d_{k}}
$$

More details can be found in [3]. The search direction $d_{k}$ generated by.

$$
B_{k} d_{k}+g_{k}=0
$$

where $B_{k}$ is an approximation of the Hessian matrix [4]. In this manuscript, we pay attention to the BFGS method in which $B_{k}$ is updated by (5).

$$
B_{k+1}^{B F G S}=B_{k}-\frac{B_{k} s_{k} s_{k}^{T} B_{k}^{T}}{s_{k}^{T} B_{k} s_{k}}+\frac{y_{k} y_{k}^{T}}{s_{k}^{T} y_{k}}
$$

where $s_{k}=x_{k+1}-x_{k}=\alpha_{k} d_{k}$ and $y_{k}=g_{k+1}-g_{k}$. Consider $H_{k}$ be the inverse of $B_{k}$. Surely, the (5) update can be written as (6).

$$
H_{k+1}^{B F G S}=H_{k}-\frac{H_{k} y_{k} s_{k}^{T}+s_{k} y_{k}^{T} H_{k}}{s_{k}^{T} y_{k}}+\left[1+\frac{y_{k}^{T} H_{k} y_{k}}{s_{k}^{T} y_{k}}\right] \frac{s_{k} s_{k}^{T}}{s_{k}^{T} y_{k}}
$$

To the best of our knowledge the BFGS method may fail to converge by using Wolfe line search for non-convex optimization and also by using with exact line search constructed Dai [2] and Mascarenhas [5]. To defeat (remedy) this case, quasi-Newton equation has been modified in some way or other can be seen some formulas among others as.

\begin{tabular}{lcc}
\hline \multicolumn{1}{c}{ Author(s) } & QN - conditions & References \\
\hline Wei, Li, and Qi & $B_{k+1} s_{k}=\tilde{y_{k}}=y_{k}+\frac{2\left(f_{k}-f_{k+1}\right)+\left(g_{k+1}+g_{k}\right)^{T} s_{k}}{s_{k}^{T} v_{k}} v_{k}$ & {$[6]$} \\
Biglari, Hassan, and Leon & $B_{k+1} s_{k}=\tilde{y}_{k}=y_{k}+\frac{4\left(f_{k}-f_{k+1}\right)+2\left(g_{k+1}+g_{k}\right)^{T} s_{k}}{s_{k}^{T} v_{k}} v_{k}$ & \\
Chen, Deng, and. Zhang & $B_{k+1} s_{k}=\tilde{y}_{k}=y_{k}+\frac{6\left(f_{k}-f_{k+1}\right)+3\left(g_{k+1}+g_{k}\right)^{T} s_{k}}{s_{k}^{T} v_{k}} v_{k}$ \\
Basim & $B_{k+1} s_{k}=\tilde{y_{k}}=\frac{1}{2} y_{k}+\frac{\left(f_{k}-f_{k+1}\right)-1 / 2\left(g_{k+1}^{T} s_{k}\right)}{s_{k}^{T} v_{k}} v_{k}$ \\
Basim and Mohammed & $B_{k+1} s_{k}=\tilde{y_{k}}=y_{k}-\frac{g_{k+1}^{T} s_{k}}{s_{k}^{T} v_{k}} v_{k}$ \\
Basim and Ghada & $B_{k+1} s_{k}=\tilde{y}=y_{k}+\frac{2\left(f_{k}-f_{k+1}\right)-s_{k}^{T} y_{k}}{s_{k}^{T} v_{k}}$
\end{tabular}

where $v_{k}$ is any vector satisfying $s_{k}^{T} v_{k} \neq 0$. In [12], a general framework of modified methods is proposed to solve non-convex optimization problems. Thus the modifying methods can guarantee the updated matrix is positive definite which guarantees a descent direction. The convergent properties are also established and generated more accurate second-order curvature approximations than the usual quasi-Newton updates do.

The quasi-Newton equation plays an important role, on studies some of the quasi-Newton methods. In this paper, we propose a modification to the hessian matrix Bk resulting in a new quasi-newton method based on new quasi-Newton equation. The proposed method can guarantee that the updated matrix is positive definite which guarantees a descent direction. The convergent properties are also established. More so the proposed method generates a more accurate second-order curvature approximations than the usual quasiNewton updates. 


\section{DERIVING THE NEW QUASI-NEWTON EQUATION}

Zahra and Ali [13] propose a type quasi-Newton equation of quasi-Newton methods and generate several QN directions, as (7).

$$
\nabla^{2} f\left(x_{k+1}\right) s_{k}=\tilde{y_{k}}=y_{k}+\frac{5}{3} \frac{\max \left\{2\left(f_{k}-f_{k+1}\right)+\left(g_{k+1}+g_{k}\right)^{T} s_{k}, 0\right\}}{s_{k}^{T} v_{k}} v_{k}
$$

For more details can be found in [13].

So we will deriving quasi-Newton equation motivated by quasi-Newton (7). Now multiplying it by $s_{k}^{T}$, we have.

$$
s_{k}^{T} \nabla^{2} f\left(x_{k+1}\right) s_{k}=s_{k}^{T} \tilde{y}_{k}=s_{k}^{T} y_{k}+\frac{5}{3}\left\{2\left(f_{k}-f_{k+1}\right)+\left(g_{k+1}+g_{k}\right)^{T} s_{k}\right\}
$$

we can write:

$$
\frac{3}{5} s_{k}^{T} \nabla^{2} f\left(x_{k+1}\right) s_{k}=\frac{3}{5} s_{k}^{T} y_{k}+2\left(f_{k}-f_{k+1}\right)+\left(g_{k+1}+g_{k}\right)^{T} s_{k}
$$

Now, from (3) and (9), we obtain.

$$
\begin{aligned}
& s_{k}^{T} \nabla^{2} f\left(x_{k+1}\right) s_{k}=\frac{3}{5} s_{k}^{T} y_{k}+2\left(f_{k}-f_{k+1}\right)+\left(g_{k+1}+g_{k}\right)^{T} s_{k}+\frac{2}{5} s_{k}^{T} \nabla^{2} f\left(x_{k+1}\right) s_{k} \\
& s_{k}^{T} \nabla^{2} f\left(x_{k+1}\right) s_{k}=\frac{3}{5} s_{k}^{T} y_{k}+2\left(f_{k}-f_{k+1}\right)+\left(g_{k+1}+g_{k}\right)^{T} s_{k}-\frac{2}{5} g_{k}^{T} s_{k}
\end{aligned}
$$

From (10) we get.

$$
s_{k}^{T} \nabla^{2} f\left(x_{k+1}\right) s_{k}=\frac{3}{5} s_{k}^{T} y_{k}+2\left(f_{k}-f_{k+1}\right)+g_{k+1}^{T} s_{k}+\frac{3}{5} g_{k}^{T} s_{k}
$$

Consider $B_{k+1}$ is an approximate of the Hessian matrix $\nabla^{2} f\left(x_{k+1}\right)$ of the $f\left(x_{k}\right)$.

$$
s_{k}^{T} B_{k+1} s_{k}=\frac{3}{5} s_{k}^{T} y_{k}+2\left(f_{k}-f_{k+1}\right)+g_{k+1}^{T} s_{k}+\frac{3}{5} g_{k}^{T} s_{k}
$$

This gives a new quasi-Newton equation in the form.

$$
B_{k+1} s_{k}=y_{k}^{*}=\frac{3}{5} y_{k}+\frac{2\left(f_{k}-f_{k+1}\right)+g_{k+1}^{T} s_{k}+3 / 5 g_{k}^{T} s_{k}}{s_{k}^{T} v_{k}} v_{k}
$$

where $v_{k}$ is any vector satisfying $s_{k}^{T} v_{k} \neq 0$. Motivated by the idea of Zahra and Ali [8], we propose a modified above quasi-Newton equation, we get as.

$$
B_{k+1} s_{k}=y_{k}^{*}=\frac{3}{5} y_{k}+\frac{\max \left\{2\left(f_{k}-f_{k+1}\right)+g_{k+1}^{T} s_{k}+3 / 5 g_{k}^{T} s_{k}\right\}}{s_{k}^{T} v_{k}} v_{k}
$$

Obviously two choices for $v_{k}$ can be computed as.

1. First case $v_{k}=g_{k+1}$ gives: 


$$
B_{k+1} s_{k}=y_{k}^{*}=\frac{3}{5} y_{k}+\frac{\max \left\{2\left(f_{k}-f_{k+1}\right)+g_{k+1}^{T} s_{k}+3 / 5 g_{k}^{T} s_{k}\right\}}{s_{k}^{T} g_{k+1}} g_{k+1}
$$

2. Second case $v_{k}=y_{k}$ gives:

$$
B_{k+1} s_{k}=y_{k}=\frac{3}{5} y_{k}+\frac{\max \left\{2\left(f_{k}-f_{k+1}\right)+g_{k+1}^{T} s_{k}+3 / 5 g_{k}^{T} s_{k}\right\}}{s_{k}^{T} y_{k}} y_{k}
$$

Using quasi-Newton equation in the BFGS update to give a good result. Now, we are in a situation to explain our algorithm in details.

Stage 1. Give $\varepsilon$, a initial point $x_{0} \in R^{n}$. Set $k=0$.

Stage 2. If possible test satisfies then stop.

Stage 3. Solve $B_{k} d_{k}=-g_{k}$.

Stage 4. Find $\alpha_{k}$ by using the following Wolfe conditions :

$$
\begin{aligned}
& f\left(x_{k}+\alpha_{k} d_{k}\right) \leq f\left(x_{k}\right)+\delta \alpha_{k} g_{k}^{T} d_{k} \\
& d_{k}^{T} g\left(x_{k}+\alpha_{k} d_{k}\right) \geq \sigma d_{k}^{T} g_{k}
\end{aligned}
$$

where $0<\delta<\sigma<1$.

Stage 5. Set $x_{k+1}=x_{k}+\alpha_{k} d_{k}$. Compute $y_{k}^{*}$ by (14). If $s_{k}^{T} y_{k}^{*}>0$, update $H_{k+1}$ by (6), otherwise let $H_{k+1}=H_{k}$. Set $k=k+1$ and go to Step 2 .

The major work, we introduce the positive definite property for the new quasi-Newton equation.

\section{Theorem 1.}

\section{Proof.}

Suppose that $s_{k}^{T} \dot{y}_{k}^{*}>0$. Then $B_{k+1}$ is symmetric positive definite.

Using definition $y_{k}^{*}$ and multiplying by $s_{k}^{T}$, we get:

$$
s_{k}^{T} y_{k}^{*}=\frac{3}{5} s_{k}^{T} y_{k}+2\left(f_{k}-f_{k+1}\right)+g_{k+1}^{T} s_{k}+\frac{3}{5} g_{k}^{T} s_{k}
$$

From (19), (17) and (18), we obtained.

$$
\begin{aligned}
s_{k}^{T} \ddot{y}_{k} & \geq-2 \delta g_{k}^{T} s_{k}+\frac{8}{5} \sigma g_{k}^{T} s_{k} \\
& \geq-2 \sigma g_{k}^{T} s_{k}+\frac{8}{5} \sigma g_{k}^{T} s_{k} \\
& \geq-\frac{2}{5} \sigma g_{k}^{T} s_{k}
\end{aligned}
$$

As $s_{k}^{T} g_{k}=\alpha_{k} d_{k}^{T} g_{k}<0$, then

$$
s_{k}^{T} \stackrel{*}{y_{k}}>-2 / 5 \sigma \alpha_{k} d_{k}^{T} g_{k}
$$

Denote the right side part of the inequality by $\Lambda$, then one has. 


$$
s_{k}^{T} y_{k}^{*}>\Lambda>0
$$

So $B_{k+1}$ is positive definite.

Though the $s_{k}^{T}{ }_{k}^{*}>0$, does not hold true for non-convex problems, $B_{k+1}$ may not be positive definite. It serves as a useful condition for positive definiteness of the updates consider.

$$
K=\left\{k: \frac{s_{k}^{T} y_{k}^{*}}{\left\|s_{k}\right\|^{2}} \geq \beta\left\|g_{k}\right\|^{\delta}\right\}
$$

where $\beta>0$ is constant and $\delta>0$ is bounded.

\section{CONVERGENCE ANALYSIS}

We will study the global convergence of our method. We need some assumptions. Let the level set $D=\left\{x / f(x) \leq f\left(x_{0}\right)\right\}$, with $x_{0}$ is an initial point of iterative method is restricted.

Using Lipschitz continuous; that is exist constants $L$ and $\gamma$, such that.

$$
\|\nabla f(v)-\nabla f(\omega)\| \leq L\|v-\omega\|, \quad \forall v, \omega \in D
$$

and,

$$
\|\nabla f(x)\| \leq \gamma, \forall x \in D
$$

Since $\left\{f\left(x_{k}\right)\right\}$ is a no increasing, which ensures $\left\{x_{k}\right\}$ is contained in $D$ and the existence of $x^{*}$ we have.

$$
\lim _{k \rightarrow \infty} f\left(x_{k}\right)=f\left(x^{*}\right)
$$

Moreover, from the fact that sequence $x_{k}$ is bounded, there exists $\mu$, such that $\forall k$, .

$$
\left\|s_{k}\right\|=\|x-\bar{x}\| \leq\|x\|+\|\bar{x}\| \leq \mu
$$

More details can be found in [14], [15].

Presented the useful theorem to prove that our method is globally convergent.

\section{Theorem 2.} (27) holds.

If $\|\nabla f(x)\| \leq \gamma$ is not satisfies for all $k$. Let $\left\{x_{k}\right\}$ be generated by modified methods, and the

$$
\left\|B_{k} s_{k}\right\| \leq a_{1}\left\|s_{k}\right\| \text { and } s_{k}^{T} B_{k} s_{2} \geq a_{2}\left\|s_{k}\right\|^{2},
$$

where $a_{1}>0$ and $a_{2}>0$ are constants. For infinitely $k$, then we have.

$$
\lim _{k \rightarrow \infty} \inf \left\|g_{k}\right\|=0
$$


Proof :

By (4) of the Wolfe conditions we obtain.

$$
\left(g_{k+1}-g_{k}\right)^{T} d_{k} \geq-(1-\sigma) g_{k}^{T} d_{k}
$$

By using Lipschitz condition we obtain.

$$
\left(g_{k+1}-g_{k}\right)^{T} d_{k} \leq L \alpha_{k}\left\|d_{k}\right\|
$$

This implies that.

$$
\alpha_{k} \geq \frac{-(1-\sigma) g_{k}^{T} d_{k}}{L\left\|d_{k}\right\|^{2}}=\frac{(1-\sigma) d_{k}^{T} B_{k} d_{k}}{L\left\|d_{k}\right\|^{2}} \geq \frac{(1-\sigma) a_{2}}{L}
$$

using (22) we obtain.

$$
\sum_{k=1}^{\infty}\left(f_{k}-f_{k+1}\right)=\lim _{N \rightarrow \infty} \sum_{k=1}^{\infty}\left(f_{k}-f_{k+1}\right)=\lim _{N \rightarrow \infty}\left(f_{1}-f_{k+1}\right)=f_{1}-f^{*}
$$

The (32) implies

$$
\sum_{k=1}^{\infty}\left(f_{k}-f_{k+1}\right) \leq+\infty
$$

This together with Wolfe condition (3) yields.

$$
\sum_{k=1}^{\infty}-\alpha_{k} g_{k}^{T} d_{k} \leq+\infty
$$

Then

$$
\lim _{k \rightarrow \infty} \alpha_{k} g_{k}^{T} d_{k}=0
$$

in cooperation with (31) provide that.

$$
\lim _{k \rightarrow \infty} d_{k}^{T} B_{k} d_{k}=\lim _{k \rightarrow \infty}-g_{k}^{T} d_{k}=0
$$

Combining (33) with (24) we obtain the conclusion (25). The proof is finished.

Nevertheless, we give the lemma for large scale problems to show the convergence property for new algorithm. This lemma was shown by Powell [16].

\section{Lemma 1.}

If BFGS method with Wolfe condition is applied to a continuously differentiable function $f$ that is bounded below, and if there exists a constant $M$ such that the inequality holds.

$$
\frac{\left\|y_{k}\right\|^{2}}{s_{k}^{T} y_{k}} \leq M
$$

Then, 


$$
\lim _{k \rightarrow \infty} \inf \left\|g_{k}\right\|=0
$$

\section{Theorem 3.}

Let $\left\{x_{k}\right\}$ be generated by the proposed method and $v_{k} \leq \chi\left\|s_{k}\right\|$. Then we have.

$$
\lim _{k \rightarrow \infty} \inf \left\|g_{k}\right\|=0
$$

\section{Proof:}

Using a contradiction method with there exists $\varepsilon>0$ such that.

$$
\left\|g_{k}\right\|>\varepsilon
$$

Hence, (22) imply that.

$$
s_{k}^{T} \stackrel{*}{y_{k}} \geq \beta\left\|g_{k}\right\|^{\delta}\left\|s_{k}\right\|^{2} \geq \beta \gamma^{\delta}\left\|s_{k}\right\|^{2}
$$

By the definition of $y_{k}$ can be written as.

$$
\stackrel{*}{y_{k}}=\frac{3}{5} y_{k}+\frac{\varpi_{k}}{s_{k}^{T} v_{k}} v_{k}, \quad \varpi_{k}=\max \left\{0,2\left(f_{k}-f_{k+1}\right)+g_{k+1}^{T} s_{k}+3 / 5 g_{k}^{T} s_{k}\right\}
$$

So, from (24), we get.

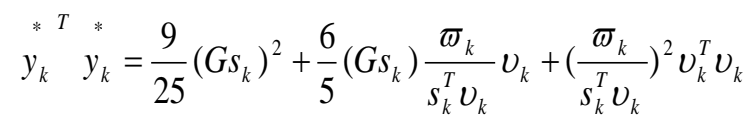

By simple computation and using $v_{k} \leq \chi\left\|s_{k}\right\|$, we obtain,

$$
\left\|y_{k}^{*}\right\|^{2} \leq s_{k}^{T}\left(\frac{3}{5} G+\chi \frac{\varpi_{k}}{s_{k}^{T} v_{k}}\right)^{2} s_{k} \leq v\left\|s_{k}\right\|^{2}
$$

Therefore, by dividing the sides of inequalitie $\left\|y_{k}^{*}\right\|^{2}$, inequalities (41) we obtain.

$$
\frac{\left\|y_{k}^{*}\right\|^{2}}{s_{k}^{T} y_{k}^{*}} \leq M
$$

Using lemma 1 , to the sub $\left\{B_{k}\right\}_{k \in K}$, obviously, clearly there exist $a_{1}$ and $a_{2}$ we get (27) for infinitely many $k$. Then theorem 2. compltes the proof.

\section{NUMERICAL REPORTS}

In this part, we details results of some numerical experiments with the new method and algorithm BFGS. We choose 30 test problems with the different dimension and initial points from literature [17]. Some enlargement was observed for class from test problems [18]-[24]. We set the parameters $\sigma_{1}=0.1, \sigma_{2}=0.9$ and $\varepsilon=10^{-5}$ in the numerical experiment. By applying law Himmeblau [25], the stop criterion is "If 
$\left|f\left(x_{k}\right)\right|>10^{-5}$, let stop $1=\left|f\left(x_{k}\right)-f\left(x_{k+1}\right)\right| /\left|f\left(x_{k}\right)\right|$; Otherwise, let stop $1=\left|f\left(x_{k}\right)-f\left(x_{k+1}\right)\right|$. For every problem, if $\left\|g_{k}\right\|<\varepsilon$ or stop $1<10^{-5}$ is satisfied, the program will be stopped". The numerical results based on IN and NF, the number of iterations and the number of function evaluations, respectively.

A comparison of the algorithms is given based on the performance profiles of Dolan and More [26]. The comparison is based on NI and NF, respectively. The plots obtained via the Dolan and More performance profiles indicate that the M2 and M3 methods perform better than the M1 method. To facilitate this, we used the following notations to represent the algorithms: M1. This is the standard BFGS method defined by (6). M2. This is the new algorithm with $v_{k}=y_{k}$ is used. M3. This is defined by (17) with using $v_{k}=g_{k+1}$. Figure 1 and Figure 2, we have found that new proposed methods best BFGS method in about $(17-53) \%$ NI and (49-51)\% NF. The new methods has good numerical results.

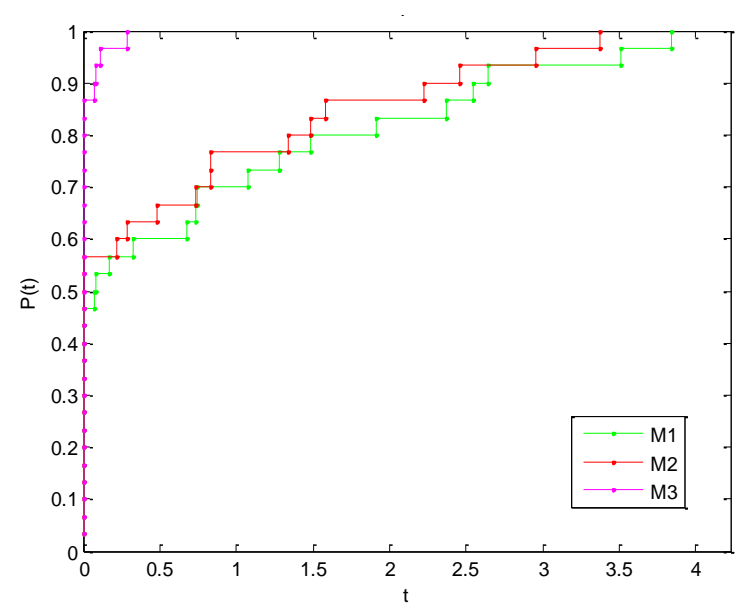

Figure 1. Number of iteration profile via dolan and more

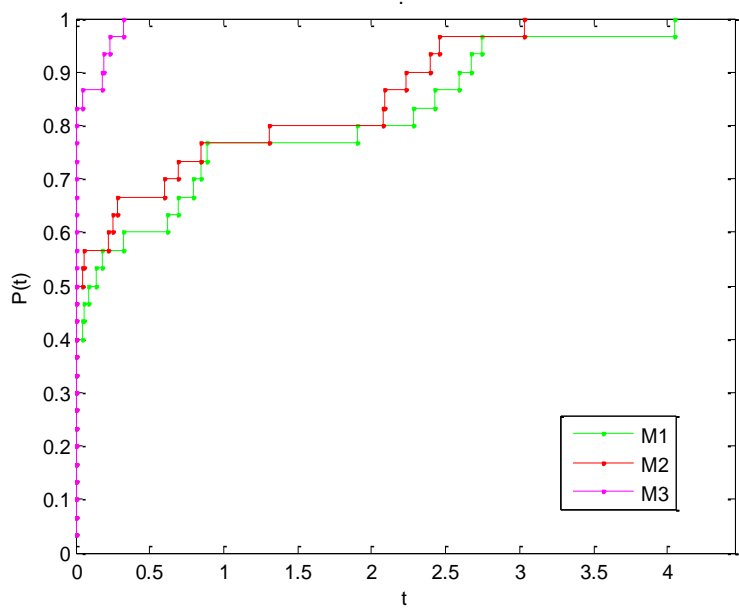

Figure 2. Number of function evaluation profile via dolan and more profile

\section{CONCLUSIONS}

The paper studies the quasi-Newton method which is popular in solving large-scale unconstrained optimization. It presents a new quasi-Newton equation for gradient methods. Numerical comparisons with the well-known BGGS method are given. Inspired new quasi-Newton very well in practice in comparison with quasi-Newton methods.

\section{ACKNOWLEDGMENT}

The first author is grateful to the University of Mosul/College of Computers Sciences and Mathematics for their provided facilities, which helped to improve the quality of this work. The second author acknowledge the financial support of her institution (Rajamangala University of Technology). The last author acknowledge with thanks, the Department of Mathematics and Applied Mathematics at the Sefako Makgatho Health Sciences University.

\section{REFERENCES}

[1] Yuan G., Sheng Z., Wang B., Hu W. and, Li C., "The global convergence of a modified BFGS method for nonconvex functions," Journal of Computational and Applied Mathematics, vol. 327, pp. 274-294, 2018, doi: 10.1016/j.cam.2017.05.030.

[2] Dai Y, "Convergence properties of the BFGS algorithm," SIAM J. Optim., vol. 13, no. 3, pp.693-701, 2013, doi: $10.1137 /$ S1052623401383455.

[3] Nocedal, J. and Wright, S. J., "Numerical optimization," Springer Series in Operations Research, 2nd edition, Springer-Verlag, New York, USA, 2006,

[4] Yuan, G, Wei, Z, and Wu, Y, "Modified limited memory BFGS method with nonmonotone line search for unconstrained optimization," J. Korean Math. Soc., vol. 47, no. 4, pp. 767-788, 2010, doi: 10.4134/JKMS.2010.47.4.767. 
[5] Mascarenhas, W.F., "The BFGS method with exact line search fails for non-convex objective functions," Mathematical Programming, vol. 99, pp. 49-61, 2004, doi: 10.1007/s10107-003-0421-7.

[6] Wei Z., Li G., and Qi L., "New quasi-Newton methods for unconstrained optimization problems," Appl. Math. Comput.,175, pp. 1156-1188, 2006, doi: 10.1016/j.amc.2005.08.027.

[7] Biglari F., Hassan M.A., and Leong W.J., "New quasi-Newton methods via higher-order tensor models," J. Comput. Appl. Math., vol. 235, no. 8, pp. 2412-242215, 2011, doi: 10.1016/j.cam.2010.10.041.

[8] Chen L.H., Deng N.Y., and. Zhang J.Z., "A modified quasi-Newton method for structured optimization with partial information on the Hessian," Comput. Optim. Appl., vol. 35, no. 1, pp. 5-18, 2006, doi: 10.1007/s10589-006-6440-6.

[9] Basim A. Hassan, "A new type of quasi-Newton updating formulas based on the new quasi-Newton equation," American Institute of Mathematical Sciences, vol. 10, no. 2, pp. 227-235, 2019, doi: 10.3934/naco.2019049.

[10] Basim A. Hassan and Ghada M., "A new quasi-Newton equation on the gradient methods for optimization minimization problem," Indonesian Journal of Electrical Engineering and Computer Science (IJEECS), vol. 19, no. 2, pp. 737-744, 2020, doi: 10.11591/ijeecs.v19.i2.pp737-744.

[11] Basim A. Hassan and Mohammed W. T., "A new variants of quasi-Newton equation based on the quadratic function for unconstrained optimization," Indonesian Journal of Electrical Engineering and Computer Science (IJEECS), vol. 19, no. 2, pp. 701-708, doi: 10.11591/ijeecs.v19.i2.pp701-708.

[12] Powell M. J. D., "Algorithms for nonlinear constraints that use lagrangian functions," Math. Program., vol. 14, no. 1, pp. 224-248, doi: 10.1007/BF01588967.

[13] Zahra K. and Ali A., "A new modified scaled conjugate gradient method for large-scale unconstrained optimization with non-convex objective function," Optimization Methods and Software, vol. 34, no. 4, pp. 783-796, 2019, doi: $10.1080 / 10556788.2018 .1457152$.

[14] Wei Z., G. Yu, G. Yuan, and Z. Lian, "The superlinear convergence of a modified BFGS-type method for unconstrained optimization," Comput. Optim. Appl., vol. 29, pp. 315-332, 2004, doi: 10.1023/B:COAP.0000044184.25410.39.

[15] Xiaowei F., Qin N. and Meilan Z., "Amodified quasi-Newton method for nonlinear equation," Journal of Computational and Applied Mathematics, vol. 328, pp. 44-58, 2018, doi: 10.1016/j.cam.2017.06.024.

[16] Powell M.J.D, "Some global convergence properties of a variable metric algorithm for minimization without exact line searches," Nonlinear Programming, SIAM-AMS Proceedings, Lemke, eds., SIAM, 1976, pp. 53-72.

[17] More J., Garbow B., and Hillstrome K., "Testing unconstrained optimization software," ACM Trans. Math. Software, vol. 7, pp. 17-41, 1981, doi: 10.1145/355934.355936.

[18] Basim A. Hassan, "A globally convergence spectral conjugate gradient method for solving unconstrained optimization problems," Raf. J. of Comp. \& Math's., vol. 10, no. 4, pp. 21-28, 2013, doi: 10.33899/csmj.2013.163543.

[19] Basim A. Hassan, "Development a special conjugate gradient algorithm for solving unconstrained minimization problems," Raf. J. of Comp. \& Math's., vol. 9, no. 1, pp. 73-84, 2012, doi: 10.33899/csmj.2012.163672.

[20] Basim A. Hassan and Hameed M. Sadiq., "A modified class of conjugate gradient algorithms based on quadratic model for nonlinear unconstrained optimization," Raf. J. of Comp \& Math., vol. 9, no. 1, pp. 25-37, 2014, doi: 10.33899/csmj.2012.163672.

[21] Basim A. Hassan and Omar M. E., "A new sufficient descent conjugate gradient method for nonlinear," Iraqi Journal of Statistical Sciences, vol. 26, pp. 12-24, 2014.

[22] Yabe, H., and Sakaiwa, N., "A new nonlinear conjugate gradient method for unconstrained optimization," Journal of the Operations Research Society of Japan, vol. 48, no. 4, pp. 284-296, 2005, doi: 10.15807/jorsj.48.284.

[23] Yasushi N. and Hideaki I., "Conjugate gradient methods using value of objective function for unconstrained optimization," Optimization Letters, vol. 6, no. 5, pp. 941-955, 2011, doi: 10.1007/s11590-011-0324-0.

[24] Dai Y.H., Han, J.Y., Liu, G.H., Sun, D.F., Yin, X. and Yuan, Y., "Convergence properties of nonlinear conjugate gradient methods," SIAM Journal on Optimization, vol. 10, no. 2, pp. 348-358, 1999, doi: $10.1137 / \mathrm{S} 1052623494268443$.

[25] Yuan Y., and Sun W., Theory and methods of optimization, Science Press of China, 1999.

[26] Dolan E. and Mor'e J.J., "Benchmarking optimization software with performance profiles," Math. Program., vol. 91, pp. 201-213, 2002, doi: 10.1007/s101070100263. 